\title{
Complete and energy blow-up in parabolic problems with nonlinear boundary conditions
}

\author{
Pavol Quittner and Aníbal Rodríguez-Bernal
}

\begin{abstract}
We study possible continuation of solutions of a nonlinear parabolic problem after the blow-up time. The nonlinearity in the equation is dissipative and blow-up is caused by the nonlinear boundary condition of the form $\partial u / \partial \nu=|u|^{q-1} u$ where $q>1$ is subcritical in $H^{1}(\Omega)$. If the dissipative term in the equation is linear then we show that blow-up of positive solutions is complete. If the dissipative term is superlinear then the solution can be continued inside the spatial domain. On the other hand, we find sufficient conditions on the nonlinearities guaranteeing that no reasonable continuation can be expected on the boundary.

2000 Mathematics Subject Classification: 35B45, 35J65.
\end{abstract}

Key words: superlinear parabolic problem, nonlinear boundary condition, a priori estimate, complete blow-up. 


\section{Introduction}

We consider the problem

$$
\left.\begin{array}{rlrl}
u_{t} & =\Delta u-a|u|^{p-1} u, & & x \in \Omega, t>0, \\
u_{\nu} & =|u|^{q-1} u, & & x \in \Gamma, t>0, \\
x, 0) & =u_{0}(x), & & x \in \Omega,
\end{array}\right\}
$$

where $a \geq 0, p \geq 1, q>1, u_{0} \in C^{2}(\bar{\Omega})$ satisfies compatibility conditions, $\Omega$ is a smoothly bounded domain in $\mathbb{R}^{n}$ and $\nu$ denotes the outer unit normal on the boundary $\Gamma:=\partial \Omega$. Under these assumptions it is well known that problem (1.1) admits a unique maximal classical solution $u=u\left(x, t ; u_{0}\right)$ and the maximal existence time of this solution $T\left(u_{0}\right)$ is either infinity or $u$ blows up at $T\left(u_{0}\right)$ in the $L_{\infty}(\Omega)$ norm. It is also known (see [7], [21], [2] and [4]) that some solutions blow-up in finite time if

$$
p<2 q-1 \quad \text { or } \quad a<q \text { and } \quad p=2 q-1 \text {, }
$$

while all solutions of (1.1) are global and bounded if

$$
p>2 q-1 \quad \text { or } \quad a>q \quad \text { and } \quad p=2 q-1 .
$$

In the limiting case, $p=2 q-1, a=q$, all positive solutions are global, unbounded and converge to a singular stationary solution provided $n=1$, [7].

Throughout this paper we will assume that $u_{0}$ is such that

$$
T\left(u_{0}\right)<\infty
$$

and we will be interested in possible weak continuation of the solution of (1.1) for $t>$ $T\left(u_{0}\right)$.

This question has been intensively studied in the case of the model problem

$$
\begin{aligned}
u_{t} & =\Delta u+u^{p}, & & x \in \Omega, t>0, \\
u & =0, & & x \in \Gamma, t>0, \\
u(x, 0) & =u_{0}(x) \geq 0, & & x \in \Omega,
\end{aligned}
$$

where $p>1$. For this, the so called proper minimal solutions are constructed as follows. Let $u_{k}, k=1,2, \ldots$, denote the solution of the approximation problem

$$
\left.\begin{array}{rlrl}
u_{t}-\Delta u & =\min \left(u^{p}, k\right), & & x \in \Omega, t>0, \\
u & =0, & & x \in \Gamma, t>0, \\
u(x, 0) & =u_{0}(x), & & x \in \Omega,
\end{array}\right\}
$$

Then $u_{k}$ exist globally, $u_{k} \nearrow \bar{u}$ pointwise as $k \rightarrow \infty$. The limit function $\bar{u}$ exists for all times (although it can take the value $\infty$ at some points at some times) and coincides with the classical solution up to time $T\left(u_{0}\right)$. 
If $p$ is subcritical in the Sobolev sense, i.e. $p<p_{S}:=(n+2) /(n-2)_{+}$, then the solution $u$ of (1.3) blows up completely in $\Omega$ at $t=T\left(u_{0}\right)$ which means that

$$
\bar{u}(x, t)= \begin{cases}u(x, t) & \text { for } x \in \Omega, t<T\left(u_{0}\right), \\ +\infty & \text { for } x \in \Omega, t>T\left(u_{0}\right)\end{cases}
$$

see [5, Theorem 1].

If $p$ is supercritical $\left(p>p_{S}\right)$ then the complete blow-up result mentioned above is true for generic initial data $u_{0}$ of (1.3), see [12], [11] and [19, Remark 18.2(iii)]. However, if, in addition, $p<1+6 /(n-10)_{+}$and $\Omega$ is a ball then there exist initial data $u_{0}$ for which $T\left(u_{0}\right)<\infty$ but the solution can be continued after $T\left(u_{0}\right)$ in a weak sense, see [11] and [15]. Moreover, under additional assumptions on $p$ and $u_{0}$, this continuation will be a classical solution of (1.1) for any $t \in\left(T\left(u_{0}\right), \infty\right) \backslash K$, where $K$ is a finite set, see [10].

For (1.1) proper minimal solutions can be constructed along the same lines. More precisely, let $u_{0} \geq 0$ and let $u_{k}$ be the solutions of the problem (1.1) with the nonlinearity $|u|^{q-1} u$ replaced by $\min \left(u^{q}, k\right)$. Then set $\bar{u}:=\lim _{k \rightarrow \infty} u_{k}$.

However, in the case of (1.1), all results on complete blow-up (known to the authors) require $n=1$ and $p=1$, see [9], [16]. Our aim is to show that complete blow-up (or impossibility of suitable weak continuation, at least) is also true for the higher dimensional case and/or $p>1$, provided the nonlinearities are subcritical. The subcriticality condition means $q<q_{S}$, where

$$
q_{S}:=\frac{n}{(n-2)_{+}}= \begin{cases}+\infty & \text { if } n \leq 2 \\ n /(n-2) & \text { if } n>2\end{cases}
$$

In fact, it is known that the exponent $q_{S}$ plays a similar role in (1.1) as the exponent $p_{S}$ for (1.3). Notice also that our assumption (1.2) implies $p \leq 2 q-1<p_{S}$ if $q<q_{S}$. Note that the conditions $p<p_{S}$ and $q<q_{S}$ (or only the former one in the case of (1.3)) imply that the problem is subcritical in $H^{1}(\Omega)$ in the sense of [3].

If $p=1$ then we obtain a full analogue of the result on complete blow-up for (1.3) which we state in the following theorem (proved in Section 2).

Theorem 1.1 Assume $u_{0} \geq 0, p=1, q<q_{S}$ and (1.2). Then $\bar{u}(x, t)=u(x, t)$ for any $x \in \bar{\Omega}, t<T\left(u_{0}\right)$, and $\bar{u}(x, t)=\infty$ for any $x \in \bar{\Omega}, t>T\left(u_{0}\right)$.

If $p>1$ and $a>0$ then the assertion in Theorem 1.1 cannot be true. In fact, for any $u_{0} \geq 0$ and any $x_{0} \in \Omega$ one can choose a small neighbourhood $D$ of $x_{0}$ such that the singular elliptic problem

$$
\left.\begin{array}{rlrl}
\Delta w & =a w^{p}, & & x \in D, \\
w & =+\infty, & & x \in \partial D,
\end{array}\right\}
$$


possesses a solution $w, w \geq u_{0}$ in $D$ (see [14], for example). Then it is easy to prove that $w(x)>\bar{u}(x, t)$ for any $x \in D, t>0$, and $\bar{u}$ is a classical solution of the equation in (1.1) in $\Omega \times[0, \infty)$, see [13]. In fact, using these arguments it can be shown that for some $C=C\left(\left\|u_{0}\right\|_{L^{\infty}(\Omega)}\right)$

$$
0 \leq \bar{u}(x, t) \leq \frac{C}{\operatorname{dist}(x, \Gamma)^{\frac{2}{p-1}}}, \quad \text { for all }(x, t) \in \Omega \times[0, \infty),
$$

see [4]. Hence complete blow-up in the sense of Theorem 1.1 is not possible. However, the question can be reformulated in terms of wether or not $\bar{u}=\infty$ on $\Gamma$ for $t>T\left(u_{0}\right)$. In this direction it was proved in [4] that for $p \geq 1$ and for each fixed point $x_{0}$ of the boundary $\Gamma$, the following is true: If the initial data are suitably large close to $x_{0}$ then there exists a time interval $[T, \tau]$, with $T \geq T\left(u_{0}\right)$, such that $\bar{u}$ is pinned to the value infinity in this time interval in a neighborhood of $x_{0}$ in $\Gamma$. Moreover, if the initial data is suitable large everywhere close to $\Gamma$, then $\bar{u}$ is pinned to infinity on the whole $\Gamma$. Note that $\tau=\infty$ provided the solution is monotonic in time, and the construction in [4] does not exclude the cases where $T>T\left(u_{0}\right)$ or $\tau<\infty$.

Here we will show that (under suitable assumptions) the proper minimal solution cannot be a weak solution of the full problem (1.1) for $t>T\left(u_{0}\right)$. Let us mention that by a weak solution of $(1.1)$ on $[0, T)$ we mean a function $u \in L_{p, l o c}\left([0, T), L_{p}(\Omega)\right)$ such that the trace of $u$ on $\Gamma$ (still denoted by $u$ ) satisfies $u \in L_{q, l o c}\left([0, T), L_{q}(\Gamma)\right)$ and the following identity is true for all $\varphi \in \mathcal{D}\left([0, T), C^{\infty}(\bar{\Omega})\right)$ :

$$
\begin{aligned}
\int_{0}^{T} \int_{\Omega}\left(u \varphi_{t}+u \Delta \varphi-a|u|^{p-1} u \varphi\right) d x d t & +\int_{0}^{T} \int_{\Gamma}\left(|u|^{q-1} u \varphi-u \varphi_{\nu}\right) d x d S \\
& +\int_{\Omega} u_{0}(x) \varphi(x, 0) d x=0 .
\end{aligned}
$$

(Here $\mathcal{D}$ means smooth functions with compact support). Of course in our proof we will show that the bad behaved term is $|u|^{q-1} u$ on the boundary.

The following theorem provides sufficient conditions guaranteeing that $\bar{u}$ is not a weak solution of (1.1) for $t>T\left(u_{0}\right)$ (see Section 3 for the proof and for related results).

Theorem 1.2 Assume $p+1 \leq 2 q, q<(n-1) /(n-2)+$ and (1.2). Then

$$
\limsup _{t \rightarrow T\left(u_{0}\right)^{-}} \int_{\Gamma}|u|^{q}(x, t) d S=\infty .
$$

Consequently, if $u_{0} \geq 0$ and $u_{t} \geq 0$ then $\int_{\Gamma} \bar{u}^{q}(x, t) d S=\infty$ for any $t>T\left(u_{0}\right)$.

In Section 4 we consider possibly sign-changing solutions of (1.1). In this case, proper minimal solutions can not be considered anymore. Therefore our non continuation result relies on energy arguments. For this, we denote by $E$ the energy functional,

$$
E(u):=\frac{1}{2} \int_{\Omega}|\nabla u|^{2} d x+\frac{a}{p+1} \int_{\Omega}|u|^{p+1} d x-\frac{1}{q+1} \int_{\Gamma}|u|^{q+1} d S .
$$


Assume that (1.2) holds true, $p+1 \leq 2 q$ and $q<q_{S}$. Since the problem (1.1) is subcritical in $H^{1}(\Omega)$ and the energy $E$ is nonincreasing along solutions of (1.1), one can easily show that

$$
\limsup _{t \rightarrow T\left(u_{0}\right)^{-}} \int_{\Gamma}|u|^{q+1}(x, t) d S=\infty .
$$

The following theorem shows that this is the dominant term in the energy.

Theorem 1.3 Assume (1.2) and let one of the following assumptions be satisfied

$$
\begin{gathered}
n=1, \quad p \leq 2 q-1, \quad a<q \text { if } p=2 q-1, \\
u_{0} \geq 0, \quad p<2 q-1, \quad q<q_{S}, \\
\quad p<q+\frac{2}{n}, \quad q<q_{S} .
\end{gathered}
$$

Then $E(u(t)) \rightarrow-\infty$ as $t \rightarrow T\left(u_{0}\right)^{-}$.

Let us mention that in the case of positive solutions this result guarantees

$$
E(\bar{u}(\cdot, t))=-\infty \quad \text { for any } t>T\left(u_{0}\right),
$$

where $\bar{u}$ is the function constructed above. In particular, $\bar{u}$ cannot become a classical solution of (1.1) for any $t>T\left(u_{0}\right)$ (cf. the result in [10] mentioned above).

The results for $n=1$ or $u_{0} \geq 0$ in Theorem 1.3 indicate that the condition $p<q+\frac{2}{n}$ in (1.11) is probably of technical nature. The technical problems stem from the fact that it is very difficult to compare the integrals appearing in (1.7) if $q<p<2 q-1$. In fact, all previous results on (1.1) using purely energy arguments were obtained under the assumption $p \leq q$ or $p \geq 2 q-1$. Notice also that the condition $p<q+\frac{2}{n}$ is automatically satisfied in the whole blow-up range $(p \leq 2 q-1)$ if $q<1+\frac{2}{n}$.

\section{Complete blow-up}

In this section we prove Theorem 1.1. The proof will be based on a modification of [5, Lemma 2.1] and recent results on a priori bounds for solutions of (1.1) in [20] and [8].

Proof of Theorem 1.1. Since $p=1$ and $q<q_{S}$, estimate [8, (1.7)] guaranteed by [8, Theorem 1.7] easily shows that

$$
T: L_{\infty}(\Omega) \rightarrow(0, \infty]: u_{0} \mapsto T\left(u_{0}\right) \text { is continuous, }
$$

cf. [18]. Fix $u_{0} \geq 0$ with $T\left(u_{0}\right)<\infty$ and assume $\bar{u} \not \equiv \infty$ for $t>T\left(u_{0}\right)$. Since $\bar{u}$ solves the integral equation

$$
\begin{gathered}
U(x, t)=\int_{\Omega} G(x, y, t) u_{0}(y) d y+\int_{0}^{t} \int_{\Omega} G(x, y, t-s) U(y, s) d x d s \\
+\int_{0}^{t} \int_{\Gamma} G(x, y, t-s) U^{q}(y, s) d S d s,
\end{gathered}
$$


where $G>0$ is the Green function of the linear problem

$$
\begin{array}{ll}
u_{t}=\Delta u-(a+1) u, & x \in \Omega, t>0, \\
u_{\nu}=0, & x \in \Gamma, t>0,
\end{array}
$$

there exists $\delta>0$ such that $\bar{u}(x, t)<\infty$ a.e. in $\Omega \times\left(0, T\left(u_{0}\right)+\delta\right)$. Now Lemma 2.1 below guarantees $T\left(\alpha u_{0}\right) \geq T\left(u_{0}\right)+\delta$ for any $\alpha \in(0,1)$ which contradicts (2.1).

The following lemma is a modification of [5, Lemma 2.1].

Lemma 2.1 Let $p, q, u_{0}$ be as in Theorem 1.1 and $0<T<\infty$. Assume $\bar{u}(x, t)<\infty$ a.e. in $\Omega \times(0, T)$. Let $\alpha \in(0,1)$. Then there exists a constant $C_{\alpha}<\infty$ such that $u\left(x, t ; \alpha u_{0}\right) \leq C_{\alpha}$ for any $x \in \bar{\Omega}$ and $t<T$.

Proof. We may assume $u_{0}>0$ in $\bar{\Omega}$. Let $V$ be the solution of the problem

$$
\begin{aligned}
V_{t} & =\Delta V-a V, & & x \in \Omega, t>0, \\
V_{\nu} & =0, & & x \in \Gamma, t>0, \\
V(x, 0) & =v_{0}(x), & & x \in \Omega,
\end{aligned}
$$

where $v_{0}:=\alpha u_{0}(x)$. Let $u_{\lambda}^{k}, \lambda \in\{1,1 / \alpha\}, k=1,2, \ldots$, be given by

$$
\begin{aligned}
\partial_{t} u_{\lambda}^{k} & =\Delta u_{\lambda}^{k}-a u_{\lambda}^{k} & & \text { in } \Omega \times(0, T), \\
\partial_{\nu} u_{\lambda}^{k} & =\left(u_{\lambda}^{k-1}\right)^{q} & & \text { on } \Gamma \times(0, T), \\
u_{\lambda}^{k}(x, 0) & =\lambda v_{0}(x), & & x \in \Omega,
\end{aligned}
$$

where $u_{\lambda}^{0}: \equiv 0$. Notice that $u_{\lambda}^{k} \in C^{2,1}(\bar{\Omega} \times(0, T))$ and that the maximum principle (see [1]) implies

$$
\left.\begin{array}{c}
0 \leq u_{\lambda}^{k} \leq u_{\lambda}^{k+1} \leq \bar{u} \\
\lambda u_{1}^{k} \leq u_{\lambda}^{k}
\end{array}\right\} \quad \text { in } \Omega \times(0, T) .
$$

From now on fix $\lambda:=1 / \alpha$ (hence $\left.u_{\lambda}^{k}(\cdot, 0)=u_{0}\right)$. For $m \in \mathbb{N}, \mu>1$, set

$$
\begin{aligned}
E_{\mu}^{m} & :=\left\{(x, t) \in\left(\Omega \times(0, T): u_{1}^{m}(x, t)>\mu V(x, t)\right\},\right. \\
g_{k}^{m}(\mu) & :=\inf _{(x, t) \in E_{\mu}^{m}} \frac{u_{\lambda}^{k}(x, t)}{u_{1}^{m}(x, t)}, \\
w(x, t) & :=u_{\lambda}^{k+1}(x, t)-g_{k}^{m}(\mu)^{q} u_{1}^{m}(x, t)+\mu\left(g_{k}^{m}(\mu)^{q}-g_{k+1}^{m}(\mu)\right) V(x, t) .
\end{aligned}
$$

Then $w \in C^{2,1}(\bar{\Omega} \times(0, T))$ and there exists $\delta=\delta(m, \mu)>0$ such that $t>\delta$ for any $(x, t) \in E_{\mu}^{m}$. For $k \geq m>1$ we have

$$
\begin{aligned}
w_{t} & =\Delta w-a w & & \text { in } E_{\mu}^{m}, \\
w & \geq g_{k+1}^{m}(\mu) u_{1}^{m}-g_{k}^{m}(\mu)^{q} u_{1}^{m}+\mu\left(g_{k}^{m}(\mu)^{q}-g_{k+1}^{m}(\mu)\right) V & & \text { in } E_{\mu}^{m}, \\
\partial_{\nu} w & =\left(u_{\lambda}^{k}\right)^{q}-\left(g_{k}^{m}(\mu) u_{1}^{m-1}\right)^{q} & & \text { on } \partial E_{\mu}^{m} \cap(\Gamma \times(0, T)),
\end{aligned}
$$


and, by (2.2),

$$
\begin{gathered}
g_{k}^{m}(\mu) \geq \lambda>1 \quad \text { if } E_{\mu}^{m} \neq \emptyset \\
\left(u_{\lambda}^{k}\right)^{q} \geq\left(g_{k}^{m}(\mu) u_{1}^{m}\right)^{q} \geq\left(g_{k}^{m}(\mu) u_{1}^{m-1}\right)^{q} \quad \text { in } E_{\mu}^{m} .
\end{gathered}
$$

Consequently,

$$
\partial_{\nu} w \geq 0 \quad \text { on } \partial E_{\mu}^{m} \cap(\Gamma \times(0, T)) .
$$

Since $u_{1}^{m}=\mu V$ on $\partial E_{\mu}^{m} \cap(\Omega \times(0, T))$ we have

$$
w \geq 0 \quad \text { on } \partial E_{\mu}^{m} \cap(\Omega \times(0, T)) .
$$

Now the maximum principle implies $w \geq 0$ in $E_{\mu}^{m}$.

The rest of the proof is the same as in the proof of [5, Lemma 2.1]: we obtain $E_{\mu}^{m}=\emptyset$ for $\mu>C^{*}:=\lambda\left(\lambda^{q-1}-1\right)^{-1 /(q-1)}$, hence

$$
u_{1}^{m}(x, t) \leq C^{*} V(x, t) \quad \text { in } \Omega \times(0, T) .
$$

Since the limit $U_{1}(x, t):=\lim _{m \rightarrow \infty} u_{1}^{m}(x, t)$ is a bounded integral solution of (1.1) with $u_{0}$ replaced by $\alpha u_{0}$, it coincides with $u\left(x, t ; \alpha u_{0}\right)$ for $t<T$. This concludes the proof.

\section{Continuation after blow-up}

In this section we prove Theorem 1.2 and some related results.

Let us first consider the linear problem

$$
\begin{aligned}
& u_{t}=\Delta u-b u, \quad x \in \Omega, \quad 0<t<T, \\
& u(x, t)=v(x, t), \quad x \in \Gamma, \quad 0<t<T, \\
& u(x, 0)=u_{0}(x), \quad x \in \Omega,
\end{aligned}
$$

where $b \geq 0$. Then we state the following regularity result, whose proof follows from [20, Lemma 3.2] (cf. also [8, Lemma 2.1]).

Lemma 3.1 Let $J:=(0, T), T \leq T_{0}, 1 \leq \rho \leq m \leq \infty, 1 \leq \kappa \leq r<\infty, 0 \leq s<1 / \kappa$ and assume

$$
\frac{1}{m}+\frac{n}{2 r}>\frac{1}{\rho}+\frac{n-1}{2 \kappa}+\frac{s}{2} .
$$

If $u_{0} \in W_{r}^{s}(\Omega)$ and $v \in L_{\rho}\left(J_{T}, L_{\kappa}(\Gamma)\right)$, then the solution $u$ of (3.1) satisfies

$$
\|u\|_{L_{m}\left(J_{T}, W_{r}^{s}(\Omega)\right)} \leq C\left(\left|u_{0}\right|_{s, r}+\|v\|_{L_{\rho}\left(J_{T}, L_{\kappa}(\Gamma)\right)}\right),
$$

where the constant $C>0$ depends only on $\rho, m, \kappa, r, s, \Omega, T_{0}, b$. 
Remark 3.2 In what follows we will repeatedly make use of the following argument. If $u$ is the solution of (1.1) and $u \in L_{\infty}\left((0, T), L_{s}(\Omega)\right)$ for some s such that

$$
p<1+\frac{2 s}{n} \quad \text { and } \quad q<1+\frac{s}{n}
$$

then the problem (1.1) is subcritical in $L_{s}(\Omega)$ and then the results in [17] or [3] imply a bound for $u$ in $L_{\infty}(\Omega \times(0, T))$.

Using this we get the following

Proposition 3.3 If $p+1 \leq 2 q, q<1+\frac{r}{n-1}$ for some $r>1$ and (1.2) holds true then

$$
\limsup _{t \rightarrow T\left(u_{0}\right)^{-}} \int_{\Gamma}|u|^{r}(x, t) d S=\infty
$$

Proof. Assume on the contrary that $u$ is the solution of (1.1) and $\int_{\Gamma}|u|^{r}(x, t) d S<C$ for any $t<T:=T\left(u_{0}\right)$. Notice that $|u| \leq z$, where $z$ is the solution of the linear problem

$$
\begin{aligned}
z_{t} & =\Delta z, & & x \in \Omega, \quad 0<t<T, \\
z(x, t) & =|u(x, t)|, & & x \in \Gamma, \quad 0<t<T, \\
z(x, 0) & =\left|u_{0}(x)\right|, & & x \in \Omega .
\end{aligned}
$$

From Lemma 3.1, with the choice $\rho:=m:=\infty, \kappa:=r, s:=0$ we get that $z \in$ $L_{\infty}\left((0, T), L_{s}(\Omega)\right)$ for $r \leq s<\frac{n}{n-1} r$. Since $|u| \leq z$, we have $u \in L_{\infty}\left((0, T), L_{s}(\Omega)\right)$ as well. Since $p+1 \leq 2 q$, we can chose $s$ such that $q<1+\frac{s}{n}<1+\frac{r}{n-1}$ which implies $p<1+\frac{2 s}{n}$. Now Remark 3.2 guarantees $u \in L_{\infty}(\Omega \times(0, T))$ which is a contradiction.

Now we can easily finish the

Proof of Theorem 1.2. First note that we can apply Proposition 3.3 with $r=q$ iff $q<\frac{n-1}{(n-2)_{+}}$.

If $u_{0} \geq 0$ and $u_{t} \geq 0$ then the property of $\bar{u}$ follows from the fact that $\bar{u}=u$ for $t<T\left(u_{0}\right)$ and $u_{k}$ (hence $\bar{u}$ ) are time increasing for $k$ large enough.

Remark 3.4 Note that for $q<q_{S}=\frac{n}{(n-2)_{+}}$we can apply Proposition 3.3 with $r=q+1$, cf. (1.8), while if $q \leq \frac{n}{(n-1)_{+}}$then we can take r arbitrarily close to 1 .

\section{Energy blow-up}

In this section we prove Theorem 1.3. In fact, the assertions of that theorem for $n=1$ or $u_{0} \geq 0$ or $p<q$ are easy consequences (or modifications) of results in [8]. On the other 
hand, the case $n>1, u_{0}$ possibly sign-changing and $q \leq p<q+\frac{2}{n}$ will require several nontrivial estimates.

We start by observing a simple situation. Note that the energy, see (1.7), satisfies the identity

$$
\int_{t_{0}}^{t_{1}} \int_{\Omega} u_{t}^{2} d x d t=E\left(u\left(t_{0}\right)\right)-E\left(u\left(t_{1}\right)\right) .
$$

Thus, assume now (1.2) and that $E(u(t))$ stays bounded as $t \rightarrow T\left(u_{0}\right)<\infty$. Then (4.1) implies

$$
\int_{0}^{T\left(u_{0}\right)} \int_{\Omega} u_{t}^{2} d x d t \leq C
$$

which moreover implies

$$
|u(t)|_{2} \leq C, \quad t \in\left(0, T\left(u_{0}\right)\right)
$$

where $|\cdot|_{2}$ denotes the norm in $L^{2}(\Omega)$. Consequently,

$$
u^{*}:=\lim _{t \rightarrow T\left(u_{0}\right)^{-}} u(t)
$$

exists in $L^{2}(\Omega)$. If $p+1 \leq 2 q$ and $q<1+\frac{2}{n}$ then the problem is subcritical in $L^{2}(\Omega)$, see [3], and then solving (1.1) with initial data $u^{*} \in L^{2}(\Omega)$ shows that the classical solution $u$ can be prolongated beyond $T\left(u_{0}\right)$, which is absurd. Hence, $E(u(t)) \rightarrow-\infty$ as $t \rightarrow$ $T\left(u_{0}\right)^{-}$. More general cases require more sophisticated arguments.

Let us first introduce some notation. By $r^{\prime}:=r /(r-1)$ we denote the dual exponent to $r \in(1, \infty)$, by $c, C$ generic positive constants which may vary from step to step. The norm in the Sobolev-Slobodeckii space $W_{r}^{s}(\Omega)$ or the Lebesgue space $L_{r}(\Omega)$ will be denoted by $|\cdot|_{s, r}$ or $|\cdot|_{r}$, respectively.

In the following two lemmas we shall deal with a fixed interval $J$ and we denote by $\|\cdot\|_{a ; b, c}$ and $\|\cdot\|_{a ; \Gamma, c}$ the norm in $L_{a}\left(J, W_{c}^{b}(\Omega)\right)$ and $L_{a}\left(J, L_{c}(\Gamma)\right)$, respectively. We also denote $\|\cdot\|_{a ; c}:=\|\cdot\|_{a ; 0, c}$. In what follows we will assume

$$
p<q+\frac{2}{n}, \quad q<q_{S}
$$

The next two results give a quantitative estimate of the gain of regularity of the solution from the boundary to the interior.

Lemma 4.1 Assume (1.2) and (4.5). Let $Q \geq 2, s \in[0,1 /(q+1)), J=\left(0, T\left(u_{0}\right)\right)$. Then

$$
\|u\|_{Q(q+1) ; s, q+1} \leq C\left(1+\|u\|_{Q(q+1) ; \Gamma, q+1}\right)^{p} .
$$

Proof. The proof is identical to the proof of [8, Lemma 3.6]. One only has to notice that $[8,(3.20)]$ is satisfied in our situation. 
Lemma 4.2 Assume (1.2) and (4.5). Let $p \geq q$ and $A \geq 1$.

(i) If $|u(t)|_{2} \leq C_{2}<\infty$ for any $t<T\left(u_{0}\right)$ then there exist $\varepsilon, C>0$ such that

$$
\|u\|_{A(p+1) ; p+1} \leq C\left(1+\|u\|_{A(q+1) ; \Gamma, q+1}^{1-\varepsilon}\right) .
$$

(ii) Let $n \geq 2$. If $|u(t)|_{R} \leq C_{R}<\infty$ for any $t<T\left(u_{0}\right)$ and any $R<6 n /(3 n-4)$ then there exist $\varepsilon, C>0$ such that

$$
\|u\|_{A p ; p+1} \leq C\left(1+\|u\|_{A q ; \Gamma, q+1}^{1-\varepsilon}\right) .
$$

Proof. (i) Setting $m:=\rho:=A(q+1), s:=0$ and $\kappa:=q+1$ in Lemma 3.1 we obtain

$$
\|u\|_{A(q+1) ; r} \leq C\left(1+\|u\|_{A(q+1) ; \Gamma, q+1}\right),
$$

whenever

$$
q+1 \leq r<\frac{n}{n-1}(q+1)
$$

Notice that we can choose $r>p+1$ due to $p<q+2 / n$. By interpolation we get

$$
|u(t)|_{p+1} \leq|u(t)|_{r}^{\theta}|u(t)|_{2}^{1-\theta} \leq C|u(t)|_{r}^{\theta}, \quad \theta:=\frac{r}{r-2} \frac{p-1}{p+1} .
$$

If $r>p+1$ satisfies (4.10) and

$$
\theta(p+1)<q+1
$$

then the estimate $|u(t)|_{p+1}^{A(p+1)} \leq C|u(t)|_{r}^{A \theta(p+1)}$ and (4.9) imply

$$
\|u\|_{A(p+1) ; p+1} \leq C\|u\|_{A \theta(p+1) ; r}^{\theta} \leq C\|u\|_{A(q+1) ; r}^{\theta} \leq C\left(1+\|u\|_{A(q+1) ; \Gamma, q+1}^{\theta}\right),
$$

which guarantees (4.7). In order to see that we can find $r>p+1$ satisfying (4.10) and (4.11) notice that (4.11) is equivalent to

$$
r>\frac{2(q+1)}{q+2-p}
$$

and that the right hand side of this inequality is always less than the upper estimate for $r$ in (4.10) due to (4.5).

(ii) Similarly as in (4.9) we deduce

$$
\|u\|_{A q ; r} \leq C\left(1+\|u\|_{A q ; \Gamma, q+1}\right),
$$

whenever (4.10) is true. Consider $r>p+1$. If $p+1<6 n /(3 n-4)$ then (4.8) is obvious. Hence we may assume $r>p+1 \geq 6 n /(3 n-4)>R$. By interpolation we obtain

$$
|u(t)|_{p+1} \leq|u(t)|_{r}^{\theta}|u(t)|_{R}^{1-\theta} \leq C|u(t)|_{r}^{\theta}, \quad \theta:=\frac{r}{r-R} \frac{p+1-R}{p+1} .
$$


Similarly as in the proof of (i), in order to prove (4.8) we only need to find $r>p+1$ such that (4.10) is true and $p \theta<q$, or equivalently,

$$
\operatorname{pr}(p+1-R)<q(p+1)(r-R) .
$$

Obviously, it is sufficient to solve these inequalities with $R:=6 n /(3 n-4)$. Condition (4.13) can be written in the form

$$
B r>R q(p+1), \quad B:=q(p+1)-p(p+1-R) .
$$

One can easily see that $p<q+2 / n$ guarantees $B>0$. Consequently, it suffices to show

$$
\frac{n}{n-1}(q+1)>\frac{R q(p+1)}{B},
$$

cf. (4.10) and (4.14). This inequality can be written in the form

$$
R q>n(p-q)\left(q+1-\frac{R}{p+1}\right) .
$$

Since the right hand side of (4.15) is an increasing function of $p$, it is sufficient to consider $p:=q+2 / n$. With this choice of $p,(4.15)$ is equivalent to

$$
f(q):=q^{2} n(R-2)+q(R(n+2)-4(n+1))+2(R n-n-2)>0 .
$$

It is easy to see that $f(1)>0$ and $f\left(q_{S}\right)>0$ (where $f\left(q_{S}\right):=\lim _{q \rightarrow \infty} f(q)$ if $n=2$ ). Since the minimum of the quadratic function $f$ is attained at $q^{*}:=\left(3 n^{2}-8 n-8\right) /(8 n)$, $q^{*} \notin\left(1, q_{S}\right)$ if $n \neq 6$ and $f\left(q^{*}\right)>0$ if $n=6$, the conclusion follows.

Now we are in a position to give the

Proof of Theorem 1.3. If (1.9) is satisfied then the assertion follows from [8, Remark 3.2]. Similarly, if (1.10) is true then we may use an obvious modification of [8, Remark 3.8(ii)].

Finally assume (1.11). If $p<q$ then the statement follows from [8, Remark 3.9]. Consequently, we may assume

$$
q \leq p<q+\frac{2}{n}
$$

Assume on the contrary that $E(u(t))$ stays bounded as $t \rightarrow T\left(u_{0}\right)$. Then (4.1) implies (4.2) and (4.3).

Fix $\varepsilon \in(0, q-1)$. Multiplying the equation in (1.1) by $u$ and integrating over $\Omega$ we obtain

$$
\begin{aligned}
\int_{\Omega} u_{t}(x, t) u(x, t) d x= & \int_{\Gamma}|u|^{q+1} d S-\int_{\Omega}|\nabla u|^{2} d x-a \int_{\Omega}|u|^{p+1} d x \\
= & -(2+\varepsilon) E(u(t)) \\
& \quad+c_{1} \int_{\Gamma}|u|^{q+1} d S+c_{2} \int_{\Omega}|\nabla u|^{2} d x-c_{3} \int_{\Omega}|u|^{p+1} d x
\end{aligned}
$$


where $c_{1}, c_{2}>0$ and $c_{3} \geq 0$. Since $E(u(t))$ is bounded and the square of the left hand side in the previous identity, integrated over $\left(0, T\left(u_{0}\right)\right)$, can be estimated by a constant due to (4.3) and (4.2), a straightforward use of Young's inequality yields

$$
\int_{0}^{T}\left(\int_{\Omega}|\nabla u|^{2} d x\right)^{2} d t+\int_{0}^{T}\left(\int_{\Gamma}|u|^{q+1} d S\right)^{2} d t \leq C\left(1+\int_{0}^{T}\left(\int_{\Omega}|u|^{p+1} d x\right)^{2} d t\right)
$$

where we have set $T=T\left(u_{0}\right)$ for simplicity.

Using (4.7) with $A:=2$ we see that the left hand side in the previous estimate is bounded. This information and (4.3) imply an estimate for $u$ in $L_{4}\left(0, T ; W_{2}^{1}(\Omega)\right)$. Interpolating between this estimate and (4.2) we obtain a bound for $u$ in $L_{\infty}\left(0, T ; L_{R}(\Omega)\right)$ for any $R<6 n /(3 n-4)_{+}$, see [6] or [18].

Set $q_{C L}:=1+\frac{6}{(3 n-4)_{+}}$and notice that $q_{C L} \leq q_{S}=\frac{n}{n-2}$. If $q<q_{C L}$ then we can chose $R$ as above such that $q<1+\frac{R}{n}$. Since $p+1 \leq 2 q$, we also have $p<1+\frac{2 R}{n}$ and Remark 3.2 yields a contradiction. Hence the proof is finished if $q<q_{C L}$. Since $q_{C L}=q_{S}$ if $n=1$ we may assume $n \geq 2$.

Now the rest of the proof is an easy modification of the proof of [20, Theorem 1.1(i)] (cf. also [8, the proof of Theorem 1.4(ii),(iii)]), whose main idea is to increase the range of $R$ above, by a bootstrap argument, up to some value such that $q<1+\frac{R}{n}$ for any given $q<$ $q_{S}$. For this it is enough, starting from $Q=2$, to obtain bounds for $u$ in $L_{2 Q}\left(0, T ; W_{2}^{1}(\Omega)\right)$ for sufficiently large $Q$ and use the bound in $W_{2}^{1}\left(0, T ; L_{2}(\Omega)\right)$ from (4.2), (4.3). In fact, by interpolation, these translates into bounds on $u$ in $L_{\infty}\left(0, T ; L_{R(Q)}(\Omega)\right)$ for some $R(Q) \rightarrow$ $\frac{2 n}{n-2}$ as $Q \rightarrow \infty$. In order to get these estimates one only has to replace the exponents $p, q$ in the proof of $[20$, Theorem 1.1(i) $]$ by $q, Q$, work with $J:=\left[0, T\left(u_{0}\right)\right)$ and make the following two modifications:

(i) In order to get the bound in $\mathbb{X}_{0}=L_{Q(q+1)}\left(J, W_{q+1}^{s}(\Omega)\right), s=(1 /(q+1))^{-}$, use Lemma 4.1 instead of [20, Lemma 3.2].

(ii) In the estimate of $\int_{J}\left|u_{t}\right|_{-d, q_{1}}^{\theta \tilde{Q} \beta^{\prime}}$ (where $q_{1}=(q+1) / q$ and $d>1 /(p+1)$ ), denote $p_{1}:=(p+1) / p$ and proceed as follows:

$$
\begin{aligned}
\int_{J}\left|u_{t}\right|_{-d, q_{1}}^{\theta \tilde{Q} \beta^{\prime}} d t & \leq C\left(1+\int_{J}\left|\gamma^{\prime}\left(|u|^{q-1} u\right)\right|_{-d, q_{1}}^{\theta \tilde{Q} \beta^{\prime}} d t+\left.\int_{J}|-a| u\right|^{p-1} u+\left.a u\right|_{-d, q_{1}} ^{\theta \tilde{Q} \beta^{\prime}} d t\right) \\
& \leq C\left(1+\left.\left.\int_{J}|| u\right|^{q-1} u\right|_{\Gamma, q_{1}} ^{\theta \tilde{Q} \beta^{\prime}} d t+\left.\left.\int_{J}|| u\right|^{p}\right|_{p_{1}} ^{\theta \tilde{Q} \beta^{\prime}} d t\right) \\
& \leq C\left(1+\int_{J}|u|_{\Gamma, q+1}^{\theta \tilde{Q} \beta^{\prime} q} d t\right),
\end{aligned}
$$

where we used the embedding $W_{q_{1}^{\prime}}^{d}(\Omega) \hookrightarrow L_{p_{1}^{\prime}}(\Omega)$ in the second step and Lemma 4.2(ii) with $A:=\theta \tilde{Q} \beta^{\prime}$ in the last step.

Acknowledgement. The first author was partially supported by Project BFM2003-03810, DGES Spain. The second author was supported by the VEGA Grant 1/0259/03 and the DFG Grant Gi 30/76-1. 


\section{References}

[1] H. Amann and P. Quittner, Semilinear parabolic equations involving measures and low regularity data, Trans. Amer. Math. Soc. 356 (2004), 1045-1119.

[2] F. Andreu, J.M. Mazón, J. Toledo and J.D. Rossi, Porous medium equation with absorption and a nonlinear boundary condition, Nonlinear Anal., TMA 49 (2002), 541-563.

[3] J. M. Arrieta, A. N. Carvalho and A. Rodríguez-Bernal, Parabolic Problems with Nonlinear Boundary Conditions and Critical Nonlinearities, J. Differ. Equations 165 (1999), 376-406.

[4] J. M. Arrieta and A. Rodríguez-Bernal, Localization near the boundary if blowup for reaction-diffusion equations with nonlinear boundary conditions, Commun. Partial Differ. Equations 29 (2004), 1127-1148.

[5] P. Baras and L. Cohen, Complete blow-up ater $T_{\max }$ for the solution of a semilinear heat equation, J. Funct. Anal. 71 (1987), 142-174.

[6] T. Cazenave and P.-L. Lions, Solutions globales d'équations de la chaleur semi linéaires, Commun. Partial Differ. Equations 9 (1984), 955-978.

[7] M. Chipot, M. Fila and P. Quittner, Stationary solutions, blow up and convergence to stationary solutions for semilinear parabolic equations with nonlinear boundary conditions, Acta Math. Univ. Comenianae 60 (1991), 35-103.

[8] M. Chipot and P. Quittner, Equilibria, connecting orbits and a priori bounds for semilinear parabolic equations with nonlinear boundary conditions, J. Dynamics Differ. Equations, 16 (2004) 91-138.

[9] M. Fila and J.-S. Guo, Complete blow-up and incomplete quenching for the heat equation with a nonlinear boundary condition, Nonlinear Anal., TMA 48 (2002), 995-1002.

[10] M. Fila, H. Matano and P. Poláčik, Immediate regularization after blow-up, SIAM J. Math. Anal., to appear.

[11] V. Galaktionov and J.L. Vázquez, Continuation of blow-up solutions of nonlinear heat equations in several space dimensions, Commun. Pure Applied Math. 50 (1997), $1-67$.

[12] A.A. Lacey and D. Tzanetis, Complete blow-up for a semilinear diffusion equation with a sufficiently large initial condition, IMA J. Appl. Math. 41 (1988), 207-215.

[13] J. López-Gómez and P. Quittner, Complete and energy blow-up in indefinite superlinear parabolic problems, Discrete Contin. Dynam. Sys., to appear. 
[14] M. Marcus and L. Véron, Uniqueness and asymptotic behavior of solutions with boundary blow-up for a class of nonlinear elliptic equations, Ann. Inst. Henri Poincaré, Analyse non linéaire 14 (1997), 237-274.

[15] W.-M. Ni, P.E. Sacks and J. Tavantzis, On the asymptotic behavior of solutions of certain quasilinear parabolic equations, J. Differ. Equations 54 (1984), 97-120.

[16] F. Quirós, J.D. Rossi and J.L.Vázquez, Complete blow-up and thermal avalanche for heat equations with nonlinear boundary conditions, Comm. Partial Differ. Equations 27 (2002), 395-424.

[17] P. Quittner, Global existence of solutions of parabolic problems with nonlinear boundary conditions, Banach Center Publ. 33 (1996), 309-314.

[18] P. Quittner, Continuity of the blow-up time and a priori bounds for solutions in superlinear parabolic problems, Houston J. Math. 29 (2003), 757-799.

[19] P. Quittner, Superlinear elliptic and parabolic problems, In: Proc. Sem. Diff. Equations (Pavlov, May 27-31, 2002), Univ. of West Bohemia in Pilsen (2003), 73-167.

[20] P. Quittner and $\mathrm{Ph}$. Souplet, Bounds of global solutions of parabolic problems with nonlinear boundary conditions, Indiana Univ. Math. J. 52 (2003), 875-900.

[21] A. Rodríguez-Bernal and Anas Tajdine, Nonlinear balance for reaction-diffusion equations under nonlinear boundary conditions: dissipativity and blow-up, J. Differ. Equations 169 (2001), 332-372. 